\title{
Mario Sousa Sánchez
}

\section{0-2017}

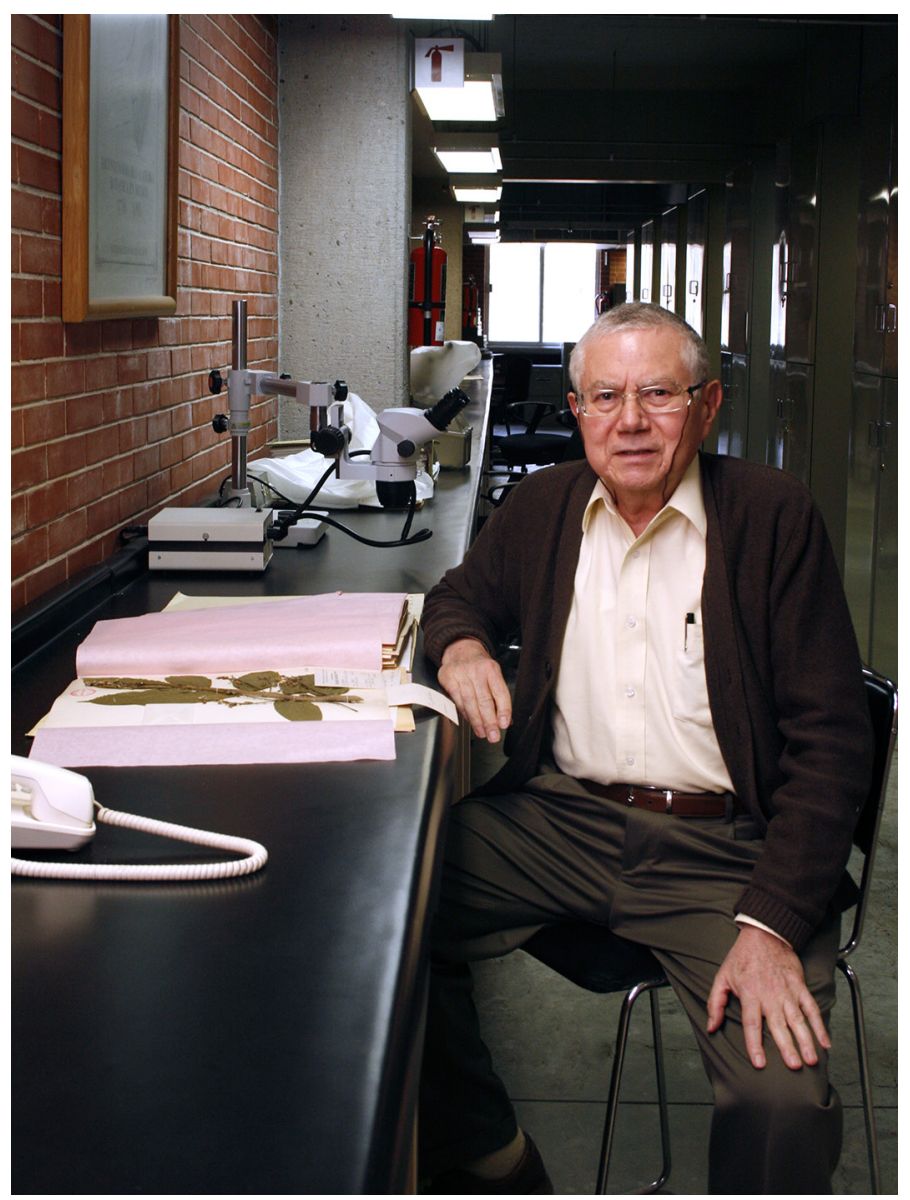

Mario Sousa Sánchez (Foto de Carmen Loyola y Fototeca IB-UNAM)

\section{Rosaura Grether González' (D)}

Mario Sousa Sánchez nació en la Ciudad de México, el 19 de febrero de 1940. Egresado de la Facultad de Ciencias de la Universidad Nacional Autónoma de México, se integró a la Comisión de Dioscóreas del Instituto de Investigaciones Forestales y realizó su tesis sobre la vegetación secundaria en la región de Tuxtepec, Oaxaca, dirigida por el Dr. Faustino Miranda, obteniendo el título de Licenciado en Biología en 1963.
Tuvo la oportunidad de seguir colaborando con el Dr. Miranda al ser contratado como Técnico Académico del Jardín Botánico de la UNAM en 1964. A manera de vivencia, Mario Sousa nos relató, en el libro dedicado a Faustino Miranda en 2007, el momento en que, como joven biólogo, habló con su maestro sobre su deseo de hacer un posgrado en el extranjero, continuando con el estudio de la vegetación tropical, a lo que el Dr. Miranda le respondió: “¿para qué salir?”, si él era quien mejor conocía el

1 Universidad Autónoma Metropolitana-Iztapalapa, Departamento de Biología, División de CBS. rogg@xanum.uam.mx DOI: http://dx.doi.org/10.21829/abm119.2017.1229

Citar como: Grether González, R. 2017. Obituario Mario Sousa Sánchez. Acta Botanica Mexicana 119: 25-32. DOI: http://dx.doi.org/10.21829/abml19.2017.1229 


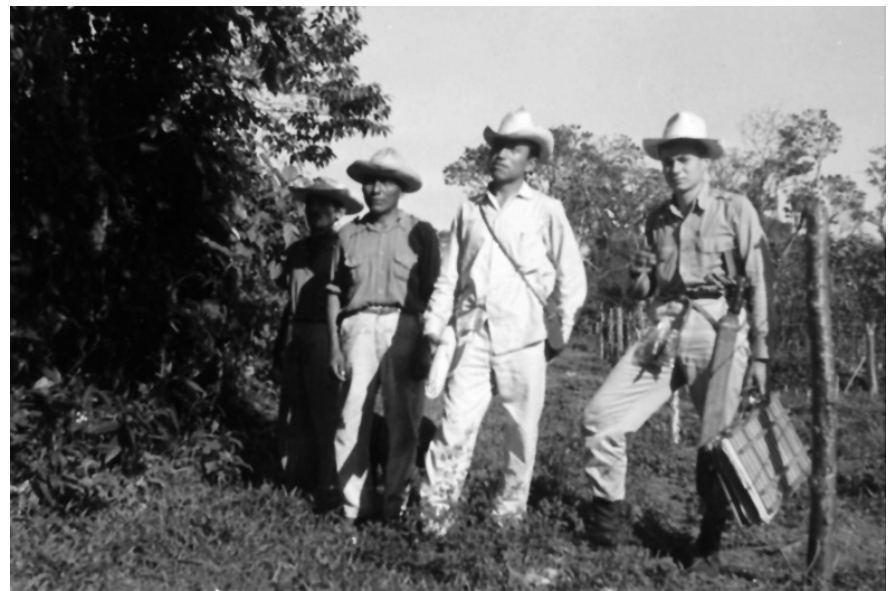

Mario Sousa Sánchez en Tuxtepec, Oaxaca, 1961 (Fototeca IB-UNAM).

tema. Mario Sousa estuvo de acuerdo con esto y, después el maestro le preguntó "si no tenía impedimento en caminar grandes trechos" a lo que respondió que desde luego no. Entonces, le propuso que estudiara la región de Los Tuxtlas, Veracruz, pero desafortunadamente, el Dr. Miranda falleció poco tiempo después.

Laborando en el Jardín Botánico, se le asignó la tarea de consecución de los primeros lotes para la Estación de Biología de Los Tuxtlas, que fueron tramitados ante el Departamento Agrario y donados en 1966. Diez años después, fungiría como asesor de la primera Estación de Biología de la UNAM.

Durante una estancia en el Herbario de la Universidad de California, en Berkeley, conoció a la Dra. Velva E. Rudd, quien influyó en él para realizar sus estudios de posgrado; desde entonces, ya mostraba un claro interés por las leguminosas y decidió viajar con su familia para iniciar su investigación sobre el género Lonchocarpus, bajo la asesoría de la Dra. Bernice G. Schubert, en la Universidad de Harvard (1968-1972). Las doctoras Rudd y Schubert colaboraron durante muchos años con Mario Sousa y sus alumnos en la taxonomía de las leguminosas mexicanas.

A su regreso de Harvard, ya como Investigador del Instituto de Biología de la UNAM, fue designado Jefe del Herbario Nacional (MEXU) en 1975, responsabilidad que asumió durante 10 años, con gran entusiasmo y dedicación, marcando un ritmo acelerado de crecimiento

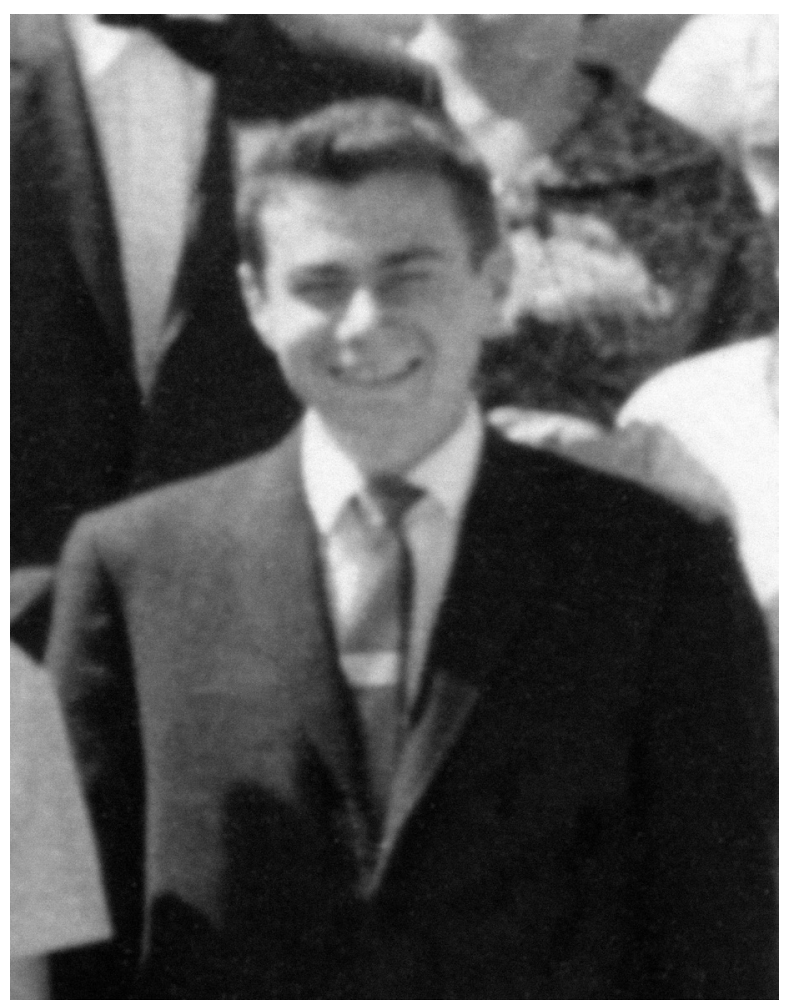

Mario Sousa Sánchez en el Segundo Congreso Mexicano de Botánica, 1963 (Fototeca IB-UNAM).

de las colecciones, que alcanzaron un total de 450,000 ejemplares, triplicando la colección existente al inicio de su gestión. Mario Sousa tuvo la cualidad de saber coordinar muy bien el trabajo realizado por todo el personal del Herbario, acercándose personalmente a los responsables de cada etapa del procesamiento de los ejemplares y cuidando la calidad de todo lo que se intercalaba en la colección. Bajo su dirección, los programas de colecta, de intercambio, de préstamos y de especialistas visitantes hicieron del Herbario Nacional uno de los más activos de Latinoamérica.

Mario Sousa trabajaba en equipo y celebraba las metas alcanzadas en el incremento de la colección, haciendo un agradable convivio con todo el personal, cada vez que se incorporaban 100,000 ejemplares más. En un segundo período como Jefe del Herbario Nacional (19942003), alcanzó la meta de superar el millón de ejemplares de plantas vasculares en la colección y tuvo la gran alegría de diseñar, junto con los ingenieros, el nuevo edificio 


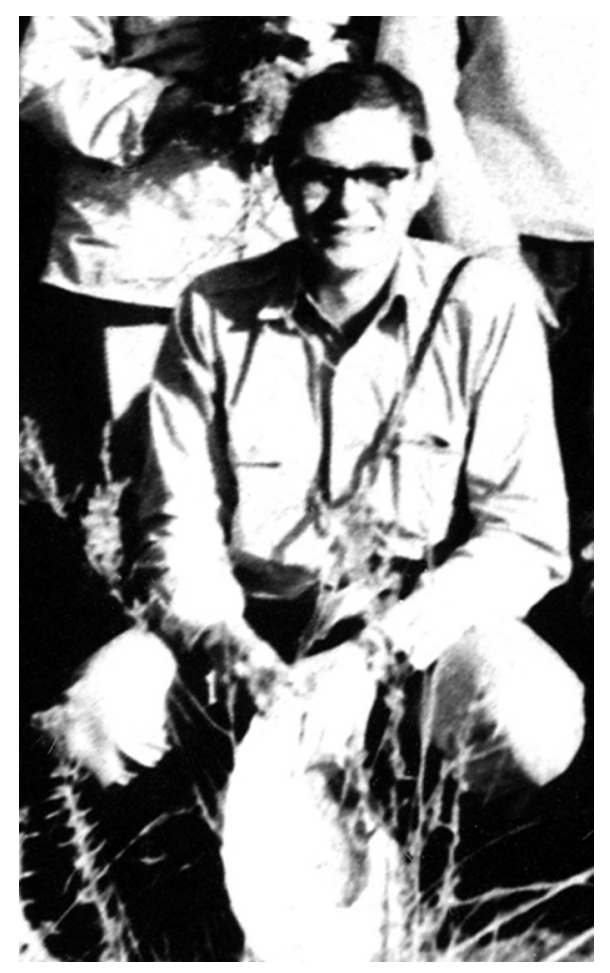

Mario Sousa Sánchez en el Tercer Congreso Mexicano de Botánica, 1966 (Fototeca IB-UNAM).

del Herbario Nacional, que abrió sus puertas en el año 1998, siendo una gran atracción para los participantes del Congreso Latinoamericano de Botánica, ya que albergaba esta enorme colección de plantas mexicanas, principalmente, y contaba con espacio para su crecimiento a futuro. En estas nuevas instalaciones, su lugar de trabajo favorito era la Sala de Tipos, donde se le encontraba diariamente revisando sus ejemplares, describiendo nuevas especies, en ocasiones concentrado y distante y en otras con una chispa de buen humor o bien, con una sonrisa amable, feliz de identificar cualquier leguminosa que le llevaran sus colegas, los alumnos y los visitantes de muy diversas instituciones.

El trabajo de campo también le gustaba mucho, planeaba cuidadosamente los itinerarios y el objetivo era lograr excelentes ejemplares botánicos, sin importar la dificultad para conseguirlos, ni la lluvia, ni la falta de la luz. En una ocasión nos llevó con lámpara en mano, al anochecer, hasta encontrar un bejuco de Mimosa watsonii en plena floración. Tampoco importaba la comida; si llevábamos víveres, él protestaba porque quitábamos espacio necesario para las plantas a prensar durante la jornada de campo. Junto con sus alumnos y colaboradores, colectó más de 13,800 números, principalmente de la familia de las leguminosas y sobretodo del sur y sureste de México, además de varios países centroamericanos. Si se consideran los duplicados de cada colecta, enviados en intercambio, suman alrededor de 55,000 ejemplares, en su mayoría de leguminosas de México y de otros países, lo que significa una importante contribución a la colección del Herbario Nacional.

El Maestro Sousa fue un notable impulsor de la exploración y la investigación botánica en México. Formó un grupo de jóvenes colectores, entrenados directamente en el campo, con todo el rigor para trabajar en los sitios más conservados, sin omitir algún elemento relevante de la flora, entre ellos están Esteban Martínez, José Carmen Soto, Arturo Solís y Alberto Reyes. Promovió la investigación botánica en muy diversas líneas, como la biología floral y la polinización, el desarrollo de plántulas, los estudios citogenéticos, la fitoquímica y el uso de las plantas medicinales, además de impulsar la formación de especialistas en grupos relevantes de plantas mexicanas y en estudios florísticos de áreas poco conocidas.

El gran interés de Mario Sousa por los trabajos florísticos lo llevó a publicar listados a nivel estatal o regional y a participar como contribuyente de las floras regionales en México, como la Flora Novo-Galiciana, la Flora del Valle de Tehuacán-Cuicatlán, la Flora del Bajío y de Regiones Adyacentes. Publicó el tratamiento de diversos géneros de leguminosas en la Flora de Nicaragua y completó muchos otros para el volumen de leguminosas de la Flora Mesoamericana, que constituyó uno de sus principales proyectos.

Su dedicación al trabajo editorial lo caracterizaba. Fungió como Jefe del Comité Editorial del Boletín de la Sociedad Botánica de México (1973-1979) y como editor de la serie Botánica de los Anales del Instituto de Biología (1993-1994). Destaca su participación como organizador del proyecto y editor general de la Flora Mesoamericana desde 1980, junto con Gerrit Davidse del Jardín Botánico 


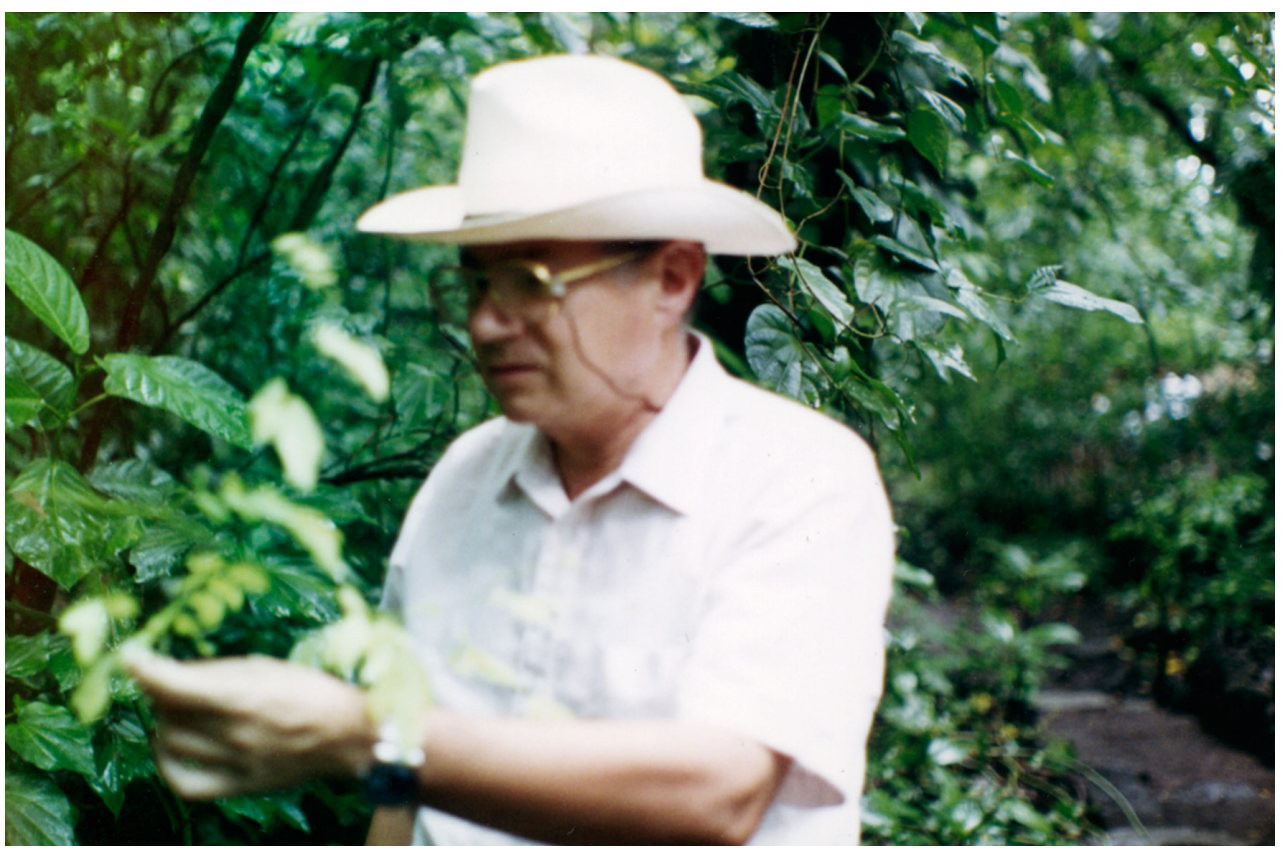

Mario Sousa Sánchez (Fototeca IB-UNAM)

de Missouri, Arthur O. Chater y Sandra Knapp del Museo de Historia Natural de Londres, y con la colaboración constante de su estimado amigo, Fernando Chiang, también como editor. Con frecuencia, Mario Sousa se encontraba revisando los manuscritos de muy diversos contribuyentes, siempre con sumo cuidado y precisión, lo que se refleja en la calidad de esta obra, que representa la Flora en castellano de mayor cobertura geográfica. Hasta el 2016, se han publicado cinco volúmenes incluyendo el tratamiento de 8186 especies.

La producción científica del Maestro Sousa incluye obras como Las colecciones botánicas de C.A. Purpus en México (1969) y el Itinerario botánico de G. Andrieux en México (1979), en las que determinó cuidadosamente las coordenadas geográficas de cada localidad, en una época en que los sistemas de información geográfica eran inexistentes. En el 2007, participó con el capítulo de Las colecciones botánicas de Faustino Miranda en el área mesoamericana de México, en colaboración con Gloria Andrade y Esteban Martínez, como parte del libro dedicado a su maestro.

$\mathrm{Su}$ valiosa aportación al conocimiento botánico comprende ca. 150 publicaciones con más de 900 citas a las mismas. Entre sus trabajos más relevantes se encuentran: el Estudio de la vegetación secundaria en la región de Tuxtepec, Oaxaca (1964), un estudio pionero en la sinecología de regiones cálido-húmedas. La subtribu Lonchocarpinae del Nuevo Mundo, en: Avances sobre Sistemática de Leguminosas (1981), en colaboración con Magdalena Peña. Leguminosae Mexicanas: Fitogeografía, endemismo y orígenes, publicado en el libro de Diversidad Biológica de México: orígenes y distribución (1993), en colaboración con Alfonso Delgado. También en 1993, publicó El género Inga (Leguminosae: Mimosoideae) del Sur de México y Centroamérica, estudio previo para la Flora Mesoamericana, que incluye 81 especies, siendo el más grande de las Mimosoideae en esa región. En colaboración con Matt Lavin publicó la Sistemática filogenética y biogeografía de la Tribu Robinieae en 1995. El capítulo de Leguminosas, en colaboración con Rosalinda Medina, Gloria Andrade y Lourdes Rico, fue publicado en el libro de Biodiversidad de Oaxaca en 2004. A lo largo de su trayectoria académica de 52 años, destaca la publicación de ca. 150 especies nuevas dentro de 29 géneros y de dos géneros nuevos para la ciencia (Apéndice 1). En 
forma adicional, hizo numerosos cambios nomenclaturales en alrededor de 20 géneros de leguminosas. La relevancia de sus trabajos y su destacada trayectoria académica lo hicieron pertenecer al Sistema Nacional de Investigadores desde 1984.

$\mathrm{Su}$ labor en la formación de recursos humanos, dedicados principalmente a la Taxonomía y Sistemática, se refleja en la dirección de 23 tesis de licenciatura y de posgrado, ocho de ellas premiadas en el Certamen de Tesis de los Congresos Mexicanos de Botánica. Esto se vincula directamente con la formación de un grupo de trabajo, que inició desde finales de los años 70, en el que se integraron muchos de sus alumnos, especializados en Taxonomía y en la familia de las leguminosas, entre ellos se encuentran Alfonso Delgado, Héctor Hernández, Lourdes Rico, Arturo Solís, Sergio Zárate, Oscar Dorado, Oswaldo Téllez, José Carmen Soto, Alberto Reyes, Rafael Torres, Ana María Hanan, Ramiro Cruz, José Linares y Rosaura Grether.

De los premios y distinciones recibidos, apreciaba mucho el haber sido nombrado Curador Honorifico del Herbario del Jardín Botánico de Missouri, la Medalla por su destacada labor en el desarrollo de la Botánica, otorgada durante la celebración del Centenario del Herbario Nacional en noviembre de 1989, la Placa en reconocimiento por su contribución al desarrollo de la Botánica en México y la incidencia de su investigación en la Península de Yucatán, en ocasión del XII Congreso Mexicano de Botánica, celebrado en Mérida, en 1993, la Medalla al Mérito Botánico, otorgada por la Sociedad Botánica de México en el XVIII Congreso Mexicano de Botánica, celebrado en Guadalajara, en 2010, entre otros. Una especial distinción fue la publicación de más de 30 especies y un género nombrados en su honor (Apéndice 2).

Mario Sousa compartió su vida y su trabajo académico con Gloria Andrade, su esposa por más de 35 años, quien colaboró en muchos de sus proyectos y lo apoyó siempre. En el 2011, su familia, colegas y amigos acompañamos a Mario Sousa en la ceremonia donde recibió el Doctorado Honoris Causa, otorgado por la Universidad Michoacana de San Nicolás de Hidalgo, en reconocimien- to a su destacada trayectoria profesional en el campo de la Botánica. Después de la emoción al recibir este merecido reconocimiento, él disfrutó mucho de una agradable cena compartiendo con todo el grupo de acompañantes en el centro de Morelia.

Otro momento de júbilo para el Dr. Sousa fue causado por el Homenaje a sus 50 años de Trayectoria Académica en el Instituto de Biología de la UNAM, celebrado en octubre de 2015. Cómo disfrutó de las conferencias, de las exposiciones y de la concurrencia de sus familiares y colegas botánicos, así como de la asistencia de muchos estudiantes de Biología.

En el XX Congreso Mexicano de Botánica, celebrado en la Ciudad de México, en septiembre de 2016, el Dr. Sousa nos habló de El impacto de la Comisión para el estudio ecológico de las Dioscóreas sobre el conocimiento de las selvas altas perennifolias mexicanas, recordando a su maestro Faustino Miranda y a sus compañeros, José Sarukhán, Miguel Angel Martínez Alfaro, Javier Chavelas, Alfredo Pérez Jiménez y Fernando Chiang, entre otros, de esa primera etapa de su trabajo botánico. Esta conferencia formó parte del Simposio "Episodios notables a través de los 75 años de la Sociedad Botánica de México".

Mario Sousa, un investigador destacado, constante en su trabajo, siempre entusiasmado por las leguminosas mexicanas, con una trayectoria académica brillante, pero sobre todo un ser humano a veces amable, otras distante, y también con buen sentido del humor, con una vida plena. Falleció en la Ciudad de México, el 17 de enero de 2017.

Ahora lo vamos a extrañar, pero tenemos motivos para celebrar una vida, la valiosa vida de un ser humano que ha contribuido de manera muy importante al conocimiento botánico, de un maestro que ha dejado escuela en los alumnos formados para continuar con la generación de conocimiento de la rica flora mexicana. Será recordado por muchos años por sus hijos Mario, Raúl, Leonardo, Gloria y María Luisa, por su esposa Gloria y por su hermana Isabel, por sus colegas, sus alumnos y sus amigos. 
Apéndice 1. Especies, subespecies, variedades, subgéneros, secciones y géneros de la familia Leguminosae descritos por Mario Sousa.

\section{Especies}

Adenopodia oaxacana M.Sousa

Calliandra erythrocephala H.M.Hern. \& M.Sousa

Calliandra physocalyx H.M.Hern. \& M.Sousa

Chamaecrista molinae G.Flores \& M.Sousa

Coursetia apantensis M.Sousa

Coursetia chiapensis Lavin \& M.Sousa

Coursetia elliptica M.Sousa \& Rudd

Coursetia oaxacensis M.Sousa \& Rudd

Coursetia paniculata M.Sousa \& Lavin

Coursetia paucifoliolata M.Sousa

Coursetia robinioides M.Sousa \& Lavin

Dalbergia agudeloi J.Linares \& M.Sousa

Dalbergia longepedunculata J.Linares \& M.Sousa

Dalbergia luteola J.Linares \& M.Sousa

Dalbergia modesta J.Linares \& M.Sousa

Dalbergia rhachiflexa J.Linares \& M.Sousa

Dalbergia ruddae J.Linares \& M.Sousa

Dalbergia salvanaturae J.Linares \& M.Sousa

Dalea schiblii Medina \& M.Sousa

Deguelia alata M.Sousa

Diphysa paucifoliolata R.Antonio \& M.Sousa

Diphysa yucatanensis Hanan-Alipi \& M.Sousa

Eriosema corymboides M.Sousa \& Fortunato

Eriosema rugosum M.Sousa \& O.Téllez

Eysenhardtia byei Cruz Durán \& M.Sousa

Eysenhardtia officinalis Cruz Durán \& M.Sousa

Galactia densiflora Germán \& M.Sousa

Guinetia tehuantepecensis L.Rico \& M.Sousa

Haematoxylum calakmulense Cruz Durán \& M.Sousa

Heteroflorum sclerocarpum M.Sousa

Hybosema robustum M.Sousa \& Lavin

Inga appendiculata M.Sousa

Inga bella $\mathrm{M}$.Sousa

Inga cabrerae M.Sousa

Inga calcicola M.Sousa

Inga chiapensis Miranda ex M.Sousa

Inga cuspidata M.Sousa

Inga dasycarpa M.Sousa

Inga davidsei M.Sousa

Inga dwyeri M.Sousa

Inga huastecana M.Sousa

Inga ismaelis M.Sousa

Inga lactifera M.Sousa

Inga lacustris M.Sousa

Inga pseudoinvolucrata M.Sousa
Inga sinacae M.Sousa \& Ibarra-Manr.

Inga tenella M.Sousa

Lonchocarpus adamsii M.Sousa

Lonchocarpus aequatorialis M.Sousa

Lonchocarpus alternifoliolatus M.Sousa

Lonchocarpus angusticarpus M.Sousa

Lonchocarpus antioquiensis M.Sousa

Lonchocarpus balsensis M.Sousa \& J.C.Soto

Lonchocarpus barbatus M.Sousa, E.Martínez \& Ramos

Lonchocarpus berriozabalensis Miranda ex M.Sousa

Lonchocarpus bicolor M.Sousa

Lonchocarpus brachyanthus M.Sousa

Lonchocarpus bracteolatus M.Sousa

Lonchocarpus branesii M.Sousa

Lonchocarpus canoensis M.Sousa

Lonchocarpus chavelasii M.Sousa

Lonchocarpus chiangii M.Sousa

Lonchocarpus congestiflorus M.Sousa \& J.Linares

Lonchocarpus crassicalyx M.Sousa

Lonchocarpus cristatus M.Sousa

Lonchocarpus epigaeus M.Sousa

Lonchocarpus ferrugineus M.Sousa

Lonchocarpus foveolatus M.Sousa

Lonchocarpus grandifoliolatus M.Sousa

Lonchocarpus guianensis M.Sousa

Lonchocarpus gyroides M.Sousa \& Cruz Durán

Lonchocarpus haberi M.Sousa

Lonchocarpus huetamoensis M.Sousa \& J.C.Soto

Lonchocarpus hughesii M.Sousa

Lonchocarpus hydrophilus M.Sousa \& J.Morales

Lonchocarpus isthmensis M.Sousa

Lonchocarpus latimarginatus M.Sousa

Lonchocarpus latisiliquus M.Sousa

Lonchocarpus linaresii M.Sousa

Lonchocarpus lomentaceus M.Sousa

Lonchocarpus longipedunculatus M.Sousa \& J.C.Soto

Lonchocarpus magallanesii M.Sousa

Lonchocarpus major M.Sousa

Lonchocarpus martinezii M.Sousa

Lonchocarpus megacarpus M.Sousa

Lonchocarpus michoacanicus M.Sousa

Lonchocarpus minor M.Sousa

Lonchocarpus monteviridis M.Sousa

Lonchocarpus monticolus M.Sousa

Lonchocarpus morenoi M.Sousa

Lonchocarpus multifoliolatus M.Sousa 
Lonchocarpus mutans M.Sousa

Lonchocarpus nebularis M.Sousa

Lonchocarpus neei M.Sousa

Lonchocarpus paucinervius M.Sousa

Lonchocarpus pedunculatus M.Sousa

Lonchocarpus pilosus M.Sousa

Lonchocarpus pittieri M.Sousa

Lonchocarpus savannicola M.Sousa

Lonchocarpus schubertiae M.Sousa

Lonchocarpus semideserti M.Sousa

Lonchocarpus septentrionalis M.Sousa

Lonchocarpus sericocarpus M.Sousa

Lonchocarpus sericophyllus M.Sousa

Lonchocarpus stenophyllus M.Sousa

Lonchocarpus subsessilifolius M.Sousa

Lonchocarpus sumiderensis M.Sousa

Lonchocarpus sylvicola M.Sousa

Lonchocarpus tenorioi M.Sousa

Lonchocarpus trinitensis M.Sousa

Lonchocarpus tuxtepecensis M.Sousa

Lonchocarpus velizii M.Sousa

Lonchocarpus verrucosus M.Sousa

Lonchocarpus vittatus M.Sousa

Lonchocarpus wendtii M.Sousa

Mucuna chiapaneca M.Sousa \& T.M.Moura

Muellera amazonica M.Sousa

Muellera burkartii M.Sousa

Muellera chocoensis M.Sousa
Nissolia ruddiae Cruz Durán \& M.Sousa

Pithecellobium campylacanthus L.Rico \& M.Sousa

Platymiscium calyptratum M.Sousa \& Klitg.

Rhynchosia delicatula O.Téllez \& M.Sousa

Styphnolobium burseroides M.Sousa, Rudd \& Medrano

Styphnolobium caudatum M.Sousa \& Rudd

Styphnolobium monteviridis M.Sousa \& Rudd

Styphnolobium parviflorum M.Sousa \& Rudd

Styphnolobium protantherum M.Sousa \& Rudd

Styphnolobium sporadicum M.Sousa \& Rudd

Swartzia mexicana M.Sousa \& R.Grether

Subespecies y variedades

Coursetia polyphylla Brandegee var. acutifolia M.Sousa \& Lavin Coursetia polyphylla Brandegee var. breviloba M.Sousa \& Lavin Lennea brunnescens Standl. var. novogalicensis Lavin \& M.Sousa Lennea viridiflora Seem. var. novogalicensis Lavin \& M.Sousa Lonchocarpus branesii M.Sousa subsp. vulcanicola M.Sousa Lonchocarpus huetamoensis M.Sousa \& J.C.Soto subsp. xochipalensis M.Sousa \& J.C.Soto

Lonchocarpus lanceolatus Benth. subsp. calciphilus M.Sousa

Lonchocarpus rugosus Benth. subsp. stipulaceus M.Sousa

\section{Géneros}

Guinetia L.Rico \& M.Sousa

Heteroflorum M.Sousa

Subgéneros y secciones

Ateleia subgen. Ruddia J. Linares \& M.Sousa

Lonchocarpus sect. Standleyi M.Sousa 
Apéndice 2. Género, especies y subespecie de varias familias de plantas y de coleópteros, dedicados a Mario Sousa.

\section{Género}

Mariosousa Seigler \& Ebinger

\section{Especies}

Acacia sousae L.Rico

Adenocalymma sousae A.H.Gentry

Aeschynomene sousae Rudd ex A.Delgado \& Sotuyo

Ageratina sousae B.L.Turner

Arachnothryx sousae Borhidi

Astragalus mario-sousae A.E.Estrada, Villareal \& Yen-Méndez

Ateleia sousae J.Linares

Begonia sousae Burt-Utley

Brongniartia sousae Dorado

Calathea sousandradeana H.Kenn. \& Ganders

Croton sousae Mart.Gord. \& Cruz Durán

Csapodya sousae Borhidi \& Reyes-García

Dalea sousae Barneby

Deppea sousae Borhidi, Mart.Cord. \& Cruz Durán

Erythrina sousae Krukoff \& Barneby

Haematoxylum sousanum Cruz Durán \& J.Jiménez Ram.

Harpalyce sousae Arroyo

Iresine sousae Zumaya, Borsch \& Flores Olv.
Lepanthes sousae Salazar \& Soto Arenas

Mimosa sousae R.Grether

Myrospermum sousanum A.Delgado \& M.C.Johnst.

Philodendron sousae Croat

Psychotria sousae Lorence \& Dwyer

Rhynchosia sousae Fortunato

Senna sousae Calvillo-Canadell \& Cevallos-Ferriz

Senna sousana H.S.Irwin \& Barneby

Solanum sousae S.Knapp

Sophora sousae Magallón-Puebla \& Cevallos-Ferriz

Symplocos sousae Almeda

Tephrosia sousae O.Téllez

Verbesina sousae J.J.Fay

Zapoteca sousae H.M.Hern. \& A.Campos

Subespecie

Leucaena lanceolata S.Watson subsp. sousae Zárate

Especies de Coleoptera, Bruchidae

Acanthoscelides sousai Johnson

Ctenocolum martiale Kingsolver \& Whitehead (hospederos:

Lonchocarpus cruentus Lundell y Lonchocarpus eriocarinalis Micheli, colectados por M. Sousa) 